\title{
基于尺寸识别和离子交换实现有机染料分离的一例阴离子型 MOF
}

\author{
何燕萍谭衍䂀张健* \\ (结构化学国家重点实验室 中国科学院福建物质结构研究所 福州 350002)
}

\begin{abstract}
摘要 在溶剂热的条件下, 利用 5 -氨基间苯二甲酸 $\left(\mathrm{H}_{2} \mathrm{aip}\right)$ 配体和硝酸铟 $\left[\mathrm{In}\left(\mathrm{NO}_{3}\right)_{3} \cdot 5 \mathrm{H}_{2} \mathrm{O}\right]$ 定向构筑了一例二维结构的阴 离子型金属有机框架材料, $\left\{\left(\mathrm{Me}_{2} \mathrm{NH}_{2}\right) \cdot\left[\operatorname{In}(\mathrm{aip})_{2}\right]\right\} \cdot x \mathrm{G}(\mathbf{1}, \mathrm{G}=$ 客体分子). 采用 $\mathrm{X}$ 射线单晶衍射、热分析仪 $(\mathrm{TGA})$ 和 $\mathrm{X}$ 射 线粉末衍射(PXRD)等方法对该化合物进行了结构表征. 经过活化后样品 1-ht 几乎不吸附氮气, 但却能有效地吸附氢 气和二氧化碳. 此外, 化合物 $\mathbf{1}$ 还可以通过离子交换和尺寸识别的方式来吸附阳离子亚甲基蓝(MB)染料, 而很难吸附 阴离子甲基橙 $(\mathrm{MO})$ 染料和分子尺寸更大的阳离子罗丹明 $\mathrm{B}(\mathrm{RhB})$ 染料, 实现水相中 $M B / M O$ 和 $M B / R h B$ 的选择性分离.
\end{abstract} 关键词 金属-有机框架材料; 晶体结构; 有机染料; 吸附; 分离

\section{An Anionic MOF for Separation of Organic Dyes via Cationic-Exchange and Size-Exclusion}

\author{
He, Yanping Tan, Yanxi Zhang, Jian* \\ (State Key Laboratory of Structural Chemistry, Fujian Institute of Research on the Structure of Matter, Chinese Academy of \\ Sciences, Fuzhou 350002)
}

\begin{abstract}
By employing the 5-aminoisophthalic acid $\left(\mathrm{H}_{2}\right.$ aip) ligand to assemble with $\mathrm{In}^{3+}$ ion, a two-dimensional (2D) anionic metal-organic framework (MOF), namely $\left\{\left(\mathrm{Me}_{2} \mathrm{NH}_{2}\right) \cdot\left[\operatorname{In}(\text { aip })_{2}\right]\right\} \bullet x \mathrm{G}(\mathbf{1}, \mathrm{G}=$ guest $)$, was solvothermally synthesized. $\mathrm{In}\left(\mathrm{NO}_{3}\right)_{3} \cdot 5 \mathrm{H}_{2} \mathrm{O}(38 \mathrm{mg}, 0.1 \mathrm{mmol})$ and $\mathrm{H}_{2}$ aip $(45 \mathrm{mg}, 0.25 \mathrm{mmol})$ are added to a mixed solvent of DMF/EtOH/ $\mathrm{H}_{2} \mathrm{O}(V: V:$ $V=2: 2: 1$ ), and then the solution was placed in a small vial. After ultrasonic diffusion for $2 \mathrm{~min}$, it is heated at $120{ }^{\circ} \mathrm{C}$ for $2 \mathrm{~d}$ to obtain yellowish sheet crystals (yield $75 \%$ based on $\mathrm{H}_{2}$ aip). 1 can be stable under different organic solvent and water. Single crystal diffraction is used to characterize its structure. 1 crystallizes in $P-1$ space group and presents a $2 \mathrm{D}$ sql net. There is weak hydrogen bonding interaction between layers, which effectively prevents the relative motion of the frameworks. The infinite layers further pack into a 3D framework in an $\mathrm{AB}$ fashion, thus generating some channels in every direction. Thermogravimetric analysis (TGA) and X-ray powder diffraction (PXRD) are used to measure its thermal stability and purity. The framework can be stable up to $350{ }^{\circ} \mathrm{C}$ after the guest molecular removed. For gas adsorption studies, 1 was heated at $150{ }^{\circ} \mathrm{C}$ for $6 \mathrm{~h}$ under a vacuum, and then the desolvated solid 1-ht is obtained. Gas sorption measurements indicate that 1-ht can hardly adsorb $\mathrm{N}_{2}\left(0.8 \mathrm{~cm}^{3} \cdot \mathrm{g}^{-1}\right)$, but except for $\mathrm{H}_{2}\left(91.2 \mathrm{~cm}^{3} \cdot \mathrm{g}^{-1}\right)$ and $\mathrm{CO}_{2}\left(44.9 \mathrm{~cm}^{3} \cdot \mathrm{g}^{-1}\right)$, which shows potential applications in $\mathrm{CO}_{2} / \mathrm{N}_{2}$ separation at $273 \mathrm{~K}$ and $101 \mathrm{kPa}$. In addition, based on ion-exchange and size-exclusion effect, compound 1 can rapidly adsorb cationic methylene blue (MB) from water in $2 \mathrm{~h}$, but except for anionic methyl orange (MO) and much larger Rhodamine B (RhB). Based on these characters, compound 1 can effectively separate MB over MO and RhB in their mixed water solution. This is a promising application for MOFs in this field. However, only a few MOFs have been investigated to adsorb organic dyes, and the selective separation of mixed dyes in water by MOFs remains rarely explored to date.

Keywords metal-organic framework; structure; organic dye; adsorption; separation
\end{abstract}

\section{1 引言}

近年来，随着纺织业、印刷业等与民生相关行业的 不断发展, 有机染料被广泛运用于各种工业中, 例如纺 织、印刷、着色、化妆品以及食品工业中. 与此同时, 现 代工业生产每天都会排出大量包含各种类型的染料废 水. 这已经成为一个极为严峻的环境问题, 对水资源甚 至生态系统造成了严重的威胁. 因此, 寻找各种可以从
工业废水中去除有机染料污染物的有效新方法引起了 化学研究工作者们的广泛兴趣. 目前, 已报道的三种有 效途径包括氧化降解法 ${ }^{[1 \sim 3]}$ 、凝絮物沉淀法 ${ }^{[4,5]}$ 和多孔材 料吸附法 ${ }^{[6]}$. 前两种方法都是通过分解有机染料并生 成新化合物的方式来消除染料, 但这些方法却无法实现 染料的循环使用, 造成资源的极大浪费. 相对而言, 多 孔材料吸附法显然在染料废水处理方面具有更多的优

\footnotetext{
*E-mail: zhj@fjirsm.ac.cn

Received September 5, 2014; published October 30, 2014.
}

Supporting information for this article is available free of charge via the Internet at http://sioc-journal.cn.

Project supported by the 973 Program (Nos. 2011CB932504 and 2012CB821705) and the National Natural Science Foundation of China (Nos. 21221001, 21403235).

项目受 973 计划(Nos. 2011CB932504, 2012CB821705)和国家自然科学基金(Nos. 21221001, 21403235)资助. 
势, 因为其可以实现染料的循环使用和回收利用. 泥 炭、木材、活性炭和其他多孔材料被广泛应用于吸附和 分离染料的废水中的染料. 然而, 除了活性炭外, 大多 数吸附剂是不可再生的. 这些吸附剂都还有一个致命的 缺点, 那就是对不同染料的吸附选择性较差. 理想的染 料分离和净化工艺要求回收的目标染料必须有较高纯 度. 因此, 探索新型的对某种特殊染料具有高选择性的 吸附材料是当前亟待解决的挑战性难题.

作为一种吸附材料, 金属一有机框架(MOFs)具有多 孔结构可控性和高比表面积等优点 ${ }^{[9]}$, 被广泛应用于新

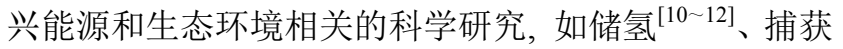
温室气体 $\left(\mathrm{CO}_{2} \text { 和 } \mathrm{CH}_{4}\right)^{[13 \sim 15]}$ 和非均相催化 ${ }^{[16 ~ 18]}$ 等. 同时, MOFs 在液相中有机染料的分离和纯化也具有巨大的潜 力, 可控的孔尺寸可对不同分子尺寸的染料进行分子识 别 ${ }^{[19]}$, 同时高的比表面积有助于提升染料负载量. 然而, 只有少数 MOFs 被用于研究水相多组分染料体系中选择 性吸附、分离或降解有机染料 ${ }^{[20 ~ 25]}$. 最近本课题组报道 的一例 In-Ag-MOF 材料可以从甲基橙(MO)的水溶液中 迅速吸附 $\mathrm{MO}$, 却不能有效地吸附亚甲基蓝(MB), 并可 以在 $\mathrm{MB} / \mathrm{MO}$ 的混合水相中选择性吸附 $\mathrm{MO}$, 分离纯度 可达 $90.1 \%{ }^{[25]}$. 对于一些离子型的有机染料分子, 离子 型 MOF 可以通过离子交换的方式来实现对这些染料的 多组分吸附和分离. 然而, 目前有关这方面的研究极少 见报道 ${ }^{[20,23]}$.

本工作利用 5-氨基间苯二甲酸 $\left(\mathrm{H}_{2} \mathrm{aip}\right)$ 配体和硝酸 铟在溶剂热的条件下合成了一例 $2 \mathrm{D}$ 的阴离子型 $\mathrm{MOF}$ 材料, 化学式为 $\left\{\left(\mathrm{Me}_{2} \mathrm{NH}_{2}\right) \cdot\left[\operatorname{In}(\text { aip })_{2}\right]\right\} \bullet x \mathrm{G}(1, \mathrm{G}=$ 客体分 子), 并采用 $\mathrm{X}$ 射线单晶衍射、热分析仪和 $\mathrm{X}$ 射线粉末 衍射等方法对材料 1 进行了结构表征, 并对去客体分子 后的样品 1-ht 进行了多种气体吸附测试. 此外, 还研究 了该化合物对甲基橙、亚甲基蓝以及罗丹明 $\mathrm{B}$ 等有机染 料的负载和分离性能, 并显示出很好的分离效果.

\section{2 结果与讨论}

\section{1 化合物 1 的晶体结构描述}

化合物 1 的空间群为 $P-1$, 每一个不对称单元包括 一个采取扭曲十二面体配位构型的 In(III)中心原子, 两 个 5-氨基间苯二甲酸根 $\left(\mathrm{a} \mathrm{ip}^{2-}\right)$ 配体和一个无序的 $\left(\mathrm{Me}_{2} \mathrm{NH}_{2}\right)^{+}$阳离子, 化学式为 $\left\{\left(\mathrm{Me}_{2} \mathrm{NH}_{2}\right) \bullet\left[\operatorname{In}(\text { aip })_{2}\right]\right\} \bullet x \mathrm{G}$. 在化合物 1 中, 每个 $\mathrm{In}(\mathrm{III})$ 原子均与四个来自于不同 aip ${ }^{2-}$ 配体的羧基配位, 形成 $\mathrm{InO}_{8}$ 构型(图 1a). 每一个 aip $^{2-}$ 配体上的羧基氧原子以螯合的形式与中心 $I n(I I I)$ 原 子配位, 而氨基基团则未参与配位. 如果把每个 In(III) 中心原子看作是一个节点, 通过 $a^{2}{ }^{2-}$ 配体连接毗邻的四 个 $\mathrm{In}(\mathrm{III})$ 原子, 进而形成一个 sql 拓扑类型的 $2 \mathrm{D}$ 层(图 $1 b)$. 层与层之间存在弱的氢键作用 $[\mathrm{N}(1)-\mathrm{H}(1 \mathrm{~A}) \cdots \mathrm{O}(8)$ 和 $\mathrm{N}(2)-\mathrm{H}(2 \mathrm{~B}) \cdots \mathrm{O}(2)$ ], 如图 1c 所示. $\mathrm{N}-\mathrm{H} \cdots \mathrm{O}$ 键的距 离在 $3.1443 \sim 3.2439 \AA$ 范围内, 角度在 $148.289(8)^{\circ} \sim$
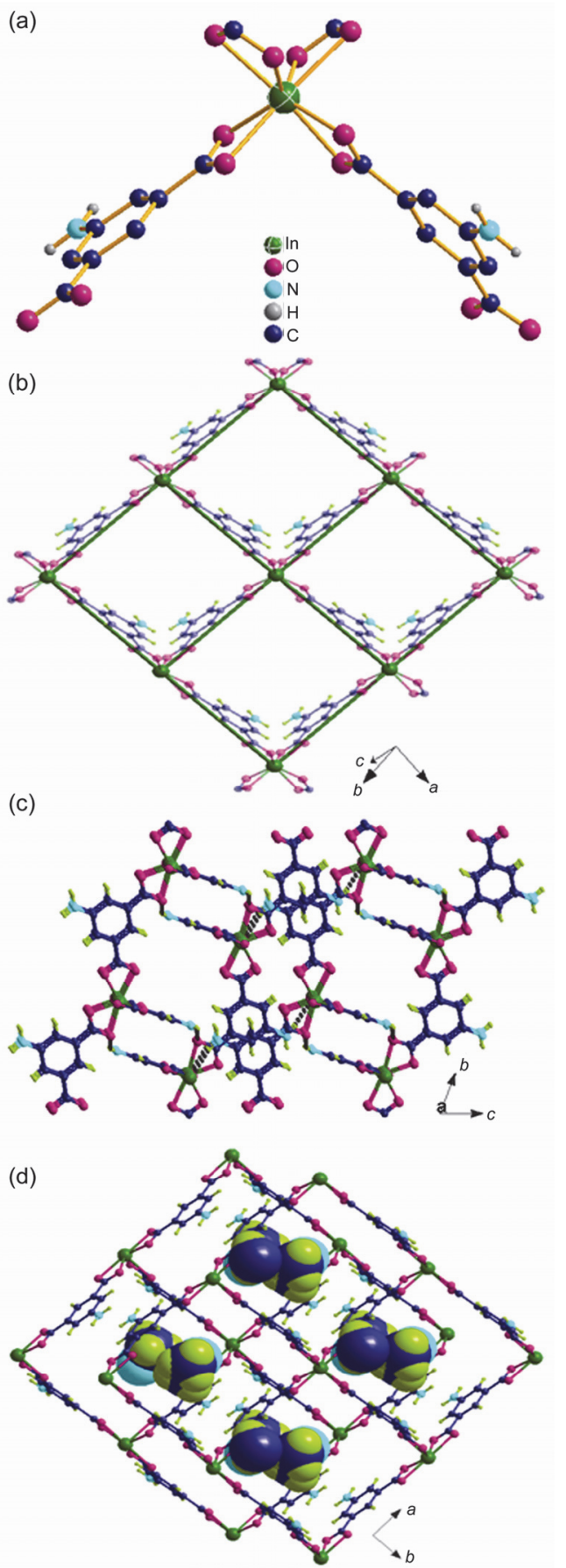

图 1 (a) In(III) 原子的配位环境图、(b) sqI 拓扑类型的 2D 层、(c)层 与层之间的氢键示意图和(d) 2D 层堆积图

Figure 1 (a) Coordination environment of $\mathrm{In}^{3+}$ atom, (b) view of the 2D sql net, (c) the hydrogen bonds interactions between 2D layers and (d) packed layers of 1

151.499(7) 之间. 层与层之间通过 $\mathrm{ABAB} \cdots$ 交错堆积的 方式形成 3D 的超分子结构(图 1d), 而氢键作用在一定 程度上加强了化合物 1 骨架的稳定性. 尽管孔道中填充 有无序的 $\left(\mathrm{Me}_{2} \mathrm{NH}_{2}\right)^{+}$阳离子, 化合物 1 仍在各个方向上 具有一定尺寸的小孔. 经 PLATON 计算, 除去客体分子 后，化合物 1 的孔穴率 $V_{\text {void }}$ 为 $21.5 \%$. 


\section{2 化合物 1 的热稳定分析与 $X$ 射线粉末衍射图}

为了测试化合物 1 的热稳定性和纯度, 我们对其进 行了热分析(TGA)和常温 $\mathrm{X}$ 射线粉末衍射(PXRD)测试. TGA 曲线显示化合物 1 共有两步的失重(图 2a). 第一步 的失重发生在 $25 \sim 150{ }^{\circ} \mathrm{C}$ 之间, 失重约 $2.42 \%$, 推测失 重的是 $\mathrm{EtOH}$ 和 $\mathrm{H}_{2} \mathrm{O}$ 客体分子; 第二步失重发生在 $150 \sim 270{ }^{\circ} \mathrm{C}$ 之间, 失重约 $13.54 \%$, 失重的是 DMF 分子. 从 $350{ }^{\circ} \mathrm{C}$ 开始, 框架开始进行化学分解, 失去 $\mathrm{aip}^{2-}$ 配体 和平衡阳离子, 到 $550{ }^{\circ} \mathrm{C}$ 时框架完全分解完毕, 得到最 终产物 $\mathrm{In}_{2} \mathrm{O}_{3}$. PXRD 测试表明化合物 1 的衍射曲线与单 晶数据模拟的曲线完全一致(图 2b), 说明化合物 1 具有 较高纯度. 为了更好地研究化合物 1 的气体吸附性能, 我们将其放在真空条件下 $150{ }^{\circ} \mathrm{C}$ 加热处理 $6 \mathrm{~h}$, 得到去 客体分子的化合物 1-ht. 化合物 1-ht 的衍射峰的位置与 原合成样品的基本一致，只是有个别峰的强度有所变化， 这表明化合物 1 在完全去除客体分子之后, 层与层之间 只是发生了微小的滑动, 这主要得益于层间氢键作用. 因此, 化合物 1-ht 具有很高的热稳定性, 为将其应用于 气体、染料的吸附和分离提供必要条件.

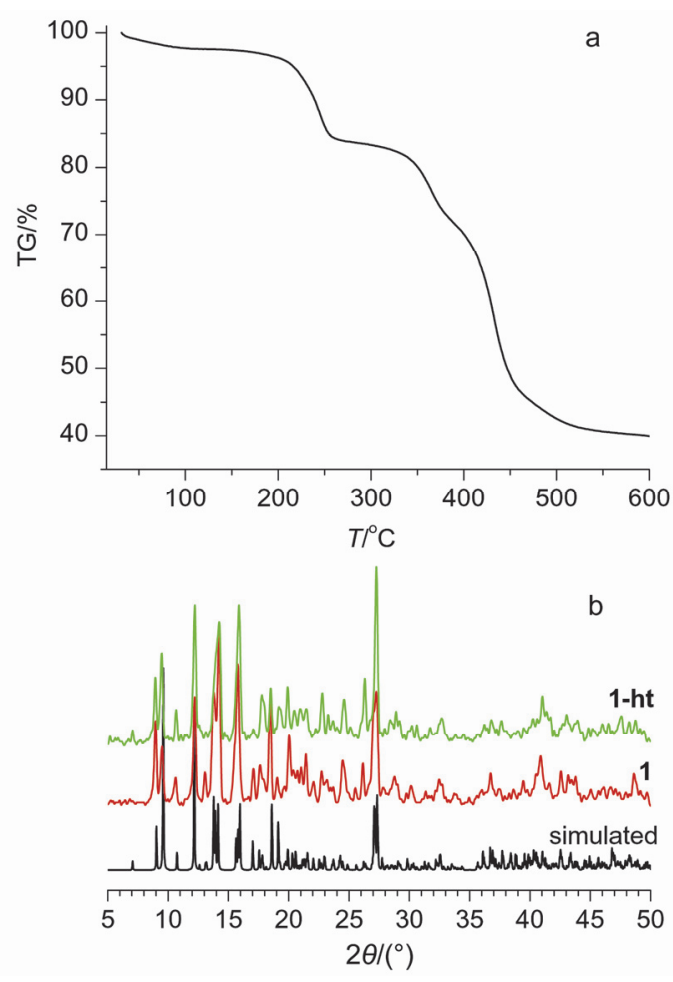

图 2 化合物 $\mathbf{1}$ 的 TGA 曲线(a)和 PXRD 图(b)

Figure 2 The TGA curve (a) and PXRD patterns (b) of 1

\section{3 化合物 1-ht 的气体吸附性能研究}

为了研究化合物 1-ht 孔结构的稳定性, 我们对其进 行了 $\mathrm{N}_{2}, \mathrm{H}_{2}$ 和 $\mathrm{CO}_{2}$ 的吸附测试. 在 $77 \mathrm{~K}$ 下, 化合物 1-ht 不能对 $\mathrm{N}_{2}$ 进行有效的吸附(图 3). 然而, 在 $77 \mathrm{~K}$ 下, 1-ht 对 $\mathrm{H}_{2}$ 吸附却属于一种典型的 type-I 吸附行为, 并且吸附 曲线与脱附曲线基本上重合, 没有出现明显的回滞环.
在 $101 \mathrm{kPa}$ 压力下, 化合物 1-ht 对 $\mathrm{H}_{2}$ 的吸附量达到了 $91.2 \mathrm{~cm}^{3} \cdot \mathrm{g}^{-1}$. 与目前报道的一些 MOFs 的储氢量相 当 ${ }^{[26 ~ 28]}$. 此外, 在 $273 \mathrm{~K}$ 下, 化合物 1-ht 对 $\mathrm{CO}_{2}$ 的吸附 量则为 $44.9 \mathrm{~cm}^{3} \cdot \mathrm{g}^{-1}$. 由此可见, 化合物 1-ht 具有潜在 的 $\mathrm{CO}_{2} / \mathrm{N}_{2}$ 分离效果.

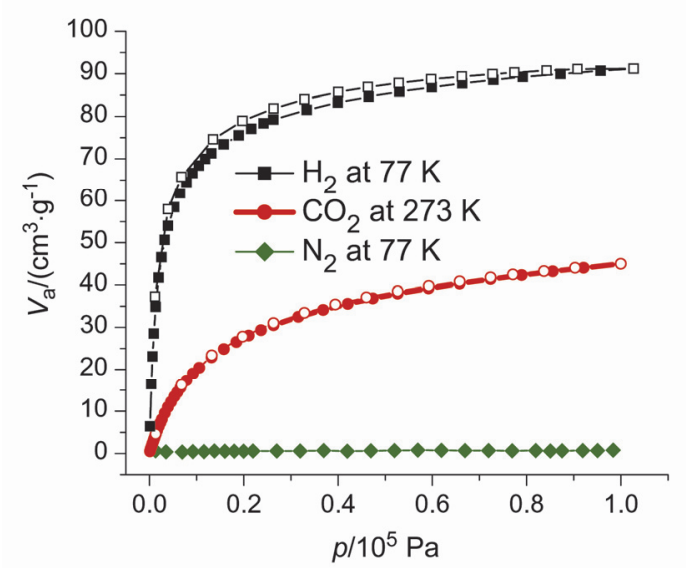

图 3 化合物 1-ht 对氮气、氢气和二氧化碳的吸附图

Figure 3 Gas adsorption isotherms of 1-ht for $\mathrm{N}_{2}, \mathrm{H}_{2}$ and $\mathrm{CO}_{2}$

\section{4 化合物 1 对染料的吸附与分离性能研究}

此外, 我们还研究了化合物 1 对甲基橙(MO)、亚甲 基蓝( $\mathrm{MB})$ 和罗丹明 $\mathrm{B}(\mathrm{RhB})$ 等有机染料的负载和分离性 能. 从图 $4 \mathrm{a}$ 可以看出, $\mathrm{MO}$ 是阴离子染料, 而 $\mathrm{MB}$ 和 $\mathrm{RhB}$ 则为阳离子染料. 试验过程中, 先称量三份 $50 \mathrm{mg}$ 固态 化合物 1 样品, 然后同时分别投入 $3 \mathrm{~mL}$ 浓度为 $40 \mathrm{mg} / \mathrm{L}$ 的 $\mathrm{MO}, \mathrm{MB}$ 和 $\mathrm{RhB}$ 的水溶液中. 如图 $4 \mathrm{~b}$ 所示, $\mathrm{MO}$ 仅是 负载于化合物 1 晶体外表面, 而化合物 1 却能有效地吸 附 MB. 这主要是因为 $\mathrm{MB}$ 中的阳离子与化合物 1 骨架 中的二甲胺阳离子进行了离子交换. 虽然 $\mathrm{RhB}$ 同为阳 离子染料, 但是由于其分子尺寸远大于化合物 1 骨架的 孔道尺寸, 不利于进行阳离子交换. 紫外-可见分光光 度计测试结果表明, 浸渍 $4 \mathrm{~h}$ 后, MO, MB 和 $\mathrm{RhB}$ 在水溶 液中的浓度分别降低至 35, 0.1 和 $27.5 \mathrm{mg} / \mathrm{L}$ (图 4b). 由 此可见, 化合物 1 对 $\mathrm{MB}$ 具有高度的选择性, 可用于回 收废水中的 MB 染料. 负载染料后的化合物 1-MO, 1-MB 和 1-RhB 的 PXRD 图谱与化合物 1 几乎一致(图 4c), 说明化合物 $\mathbf{1}$ 在水溶液中能稳定存在, 并且其框架 不随平衡阳离子之间的交换而发生明显的变化.

根据以上实验结果可以推测，化合物 $\mathbf{1}$ 可对不同离 子型的有机染料或同种离子型而尺寸不同的有机染料 进行分离. 为了验证这一推测, 两份 $50 \mathrm{mg}$ 的固态化合 物 1 分别被投于 $\mathrm{MB} / \mathrm{MO}(20 / 20 \mathrm{mg} / \mathrm{L}, 3 \mathrm{~mL})$ 和 $\mathrm{MB} / \mathrm{RhB}$ $(20 / 20 \mathrm{mg} / \mathrm{L}, 3 \mathrm{~mL})$ 混合水溶液中. 实验结果表明, 化合 物 1 可以有效地分离 $\mathrm{MB} / \mathrm{MO}$ 和 $\mathrm{MB} / \mathrm{RhB}$, 如图 5 所示.

\section{3 结论}

阴离子型 $\mathrm{MOF}$ 材料, $\left\{\mathbf{1},\left(\mathrm{Me}_{2} \mathrm{NH}_{2}\right) \cdot\left[\operatorname{In}(\text { aip })_{2}\right]\right\} \bullet x \mathrm{G}$, 
(a)<smiles>CN(C)c1ccc(N=Nc2ccc(S(N)(=O)=O)cc2)cc1</smiles>

MO<smiles>CN(C)c1ccc2nc3ccc(N(C)C)cc3[s+]c2c1</smiles>

$\mathrm{MB}$
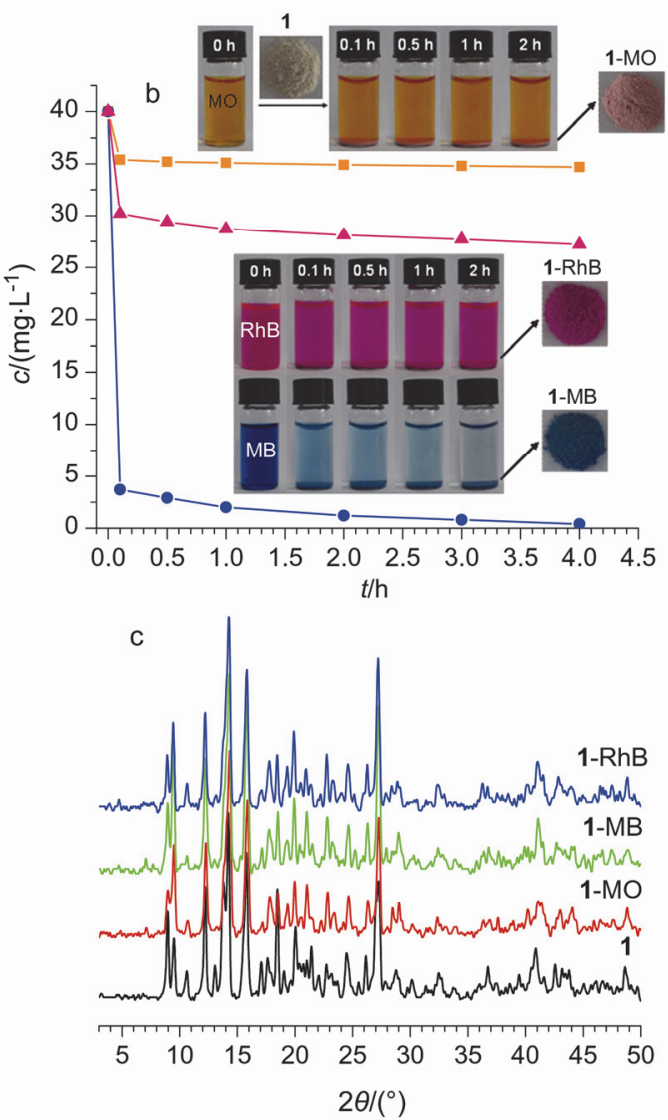

图 4 (a)甲基橙(MO)、亚甲基蓝(MB)和罗丹明 B (RhB)的结构式、(b) 化合物 1 对染料的负载过程相片和染料的浓度变化曲线和(c)化合物 1-MO, 1-MB 和 1-RhB 的粉末衍射图

Figure 4 (a) The structures of $\mathrm{MO}, \mathrm{MB}$ and $\mathrm{RhB}$, (b) photos and measured concentration curves of dyes enrichment progress over $\mathbf{1}$ in 2 hour and (c) PXRD patterns of 1-MO, 1-MB and 1-RhB

具有 $\mathbf{s q l}$ 拓扑类型的 $2 \mathrm{D}$ 层状多孔结构, 活化后可以有效 地吸附 $\mathrm{H}_{2}$ 和 $\mathrm{CO}_{2}$, 而几乎不吸附 $\mathrm{N}_{2}$. 化合物 1 还可以通 过离子交换和尺寸识别的方式来吸附阳离子亚甲基蓝 (MB), 而很难吸附阴离子甲基橙(MO)和分子尺寸较大 的阳离子罗丹明 $\mathrm{B}(\mathrm{RhB})$, 并可在双组份染料水溶液中 将 MB 选择性吸附, 达到很好的分离效果.

\section{4 实验部分}

\section{1 仪器与试剂}

小分子衍射仪, 型号 XCaliburE, Mo K $\alpha(\lambda=$

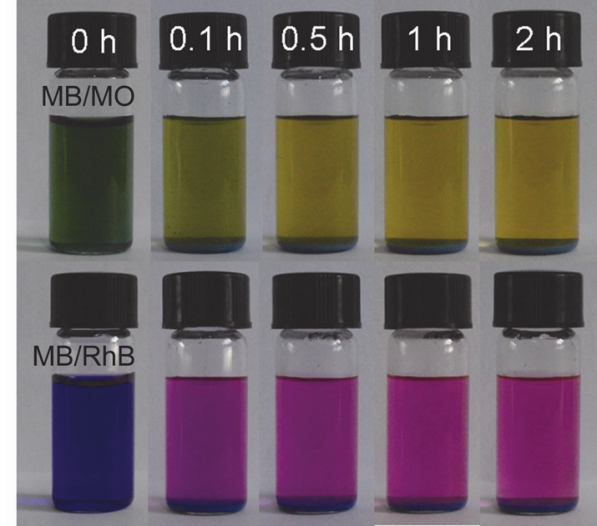

图 5 化合物 1 对 $\mathrm{MB} / \mathrm{MO}$ 或 $\mathrm{RhB}$ 的分离过程相片

Figure 5 Photos showing the selective adsorption of MB over MO or RhB by 1

$0.07107 \mathrm{~nm})$ 光源辐射; $X$ 射线粉末衍射仪, 型号 MiniFlex-II, $\mathrm{Cu} \mathrm{K \alpha}(\lambda=0.15418 \mathrm{~nm})$ 光源辐射，扫描速度 $5\left(^{\circ}\right) / \mathrm{min}$; 综合热分析仪, 型号 STA449F3, 测试环境为 氮气气氛, 升温速率为 $10{ }^{\circ} \mathrm{C} / \mathrm{min}$; 全自动比表面积分 析仪, 型号 ASAP 2020; 紫外可见分光光度计, 型号 $752 \mathrm{~N}$.

5-氨基间苯二甲酸(纯度 98\%)、硝酸铟(纯度 $98 \%$ )、 罗丹明 $\mathrm{B}($ 纯度 $99 \%$ )、亚甲基蓝(纯度 $95 \%$ )、甲基橙(纯 度 $85 \%$ ) 和 $N, N$-二甲基甲酰胺(分析纯)、乙醇(分析纯) 以及氮气(纯度 $99.999 \%$ )、氢气(纯度 $99.999 \%$ ) 和二氧化 碳(纯度 $99.9 \%$ )均是商业购买; 去离子水则为自制.

\section{2 材料的制备}

\subsection{1 化合物 1 的合成}

在 $20 \mathrm{~mL}$ 进口螺旋盖加热反应小瓶中, 将 $\mathrm{In}\left(\mathrm{NO}_{3}\right)_{3} \cdot 5 \mathrm{H}_{2} \mathrm{O}(38 \mathrm{mg}, 0.1 \mathrm{mmol})$ 溶于 $5 \mathrm{~mL}$ 的 DMF (2 $\mathrm{mL}), \mathrm{EtOH}(2 \mathrm{~mL})$ 和 $\mathrm{H}_{2} \mathrm{O}(1 \mathrm{~mL})$ 混合溶液中, 然后加入 5 -氨基间苯二甲酸(45 mg, $0.25 \mathrm{mmol}$ ), 超声波振动 20 $\min$ 以混合均匀. 最后将反应瓶放到 $120{ }^{\circ} \mathrm{C}$ 的恒温烘箱 中静置 $2 \mathrm{~d}$, 即可得到淡黄色的片状晶体. 冷却至室温 并过滤，然后用 DMF 洗涤晶体，并在室温下烘干(产率: $75 \%$ 基于配体 $\mathrm{H}_{2}$ aip).

\subsection{2 晶体的结构测定}

化合物 1 的单晶 X 射线衍射数据是利用 XCaliburE 衍射仪在常温下进行测定, 使用石墨单色器, 所用射线 为 Mo K $\alpha(\lambda=0.71073 \AA)$, 数据的还原和结构解析工作 分别使用 Bruker SHELXTL 和 SHELXTL-97 程序完成. 利用直接法确定金属原子位置, 然后用差值函数法和最 小二乘法求出全部非氢原子坐标, 最后用最小二乘法对 结构进行修正. 化合物的氢原子是理论加氢.

该化合物晶体属于三斜晶系, 空间群为 $P-1$, 晶胞 参数: $a=9.8764(5) \AA, b=9.9547(5) \AA, c=13.5467(7) \AA$, $\alpha=68.736(5)^{\circ}, \quad \beta=82.259(4)^{\circ}, \quad \gamma=87.525(4)^{\circ}, \quad V=$ 1229.86(11) $\AA^{3}, T=293(2), Z=2, D_{\mathrm{c}}=1.321 \mathrm{~g} / \mathrm{cm}^{3}$, 总共 
9258 个衍射点被收集, 4317 个是独立的 $\left(R_{\mathrm{int}}=0.0347\right)$, $R_{1}=0.0393[I>2 \sigma(I)], w R_{2}\left(F^{2}\right)=0.1058 \quad[I>2 \sigma(I)]$, $G o o F=1.021$.

\section{References}

[1] Lackey, L. W.; Mines Jr, R. O.; McCreanor, P. T. J. Hazard. Mater. 2006, $138,357$.

[2] Wang, L.; Yao, Y.-Y.; Sun, L.-J.; Lü, W.-Y.; Chen, W.-X. Acta Chim. Sinica 2013, 71, 1633. (王列, 姚玉元, 孙利杰, 吕汪洋, 陈 文兴, 化学学报, 2013, 71, 1633.)

[3] Cao, T.-T.; Luo, G.-F.; Zou, C.-Q.; Zhao, X.-R.; Li, R.-P.; Huang, Y.-P. Acta Chim. Sinica 2011, 69, 1438. (曹婷婷, 罗光富, 邹彩琼, 赵小蓉, 李瑞萍, 黄应平, 化学学报, 2011, 69, 1438.)

[4] Chen, M.-M.; Ma, W.-H.; Li, J.; Huang, Y.-P.; Zhao, J. Environ. Sci. Technol. 2004, 38, 1569.

[5] Ramirez, J.; Vicente, M.; Madeira, L. Appl. Catal. B: Environ. 2010, 98, 10.

[6] Azhar, S. S.; Liew, A. G.; Suhardy, D.; Hafiz, K. F.; Irfan, M. D. Am. J. Appl. Sci. 2005, 2, 1499.

[7] Gonzalez-Olmos, R.; Holzer, F.; Kopinke, F.; Georgi, A. Appl. Catal. A: General 2011, 398, 44

[8] Martínez, F.; Pariente, M.; Ángel, J.; Melero, J.; Rubalcaba, A. J. Chem. Technol. Biotechnol. 2012, 87, 880.

[9] Su, Z.; Fan, J.; Okamura, T.; Sun, W.-Y. Chin. J. Chem. 2012, 30, 2016.

[10] Suh, M. P.; Park, H. J.; Prasad, T. K.; Lim, D.-W. Chem. Rev. 2012, $112,782$.

[11] Zhai, Q.-G.; Lin, Q.; Wu, T.; Wang, L.; Zheng, S.-T.; Bu, X.-H.; Feng, P.-Y. Chem. Mater. 2012, 24, 2624.

[12] Pang, J.; Jiang, F.; Wu, M.; Yuan, D.; Zhou, K.; Qian, J.; Su, K.;
Hong, M. Chem. Commun. 2014, 50, 2834.

[13] Zhang, Z.-J.; Gao, W.-Y.; Wojtas, L.; Ma, S.-Q.; Eddaoudi, M.; Zaworotko, M. Angew. Chem., Int. Ed. 2012, 124, 9464.

[14] Zhou, Z.-E.; Xue, C.-Y.; Yang, Q.-Y.; Zhong, C.-L. Acta Chim. Sinica 2009, 67, 477. (周子娥, 薛春瑜, 阳庆元，仲崇立，化学学 报, 2009, 67, 477.)

[15] Jia, J.-T.; Wang, L.; Zhao, Q.; Sun, F.-X.; Zhu, G.-S. Acta Chim. Sinica 2013, 71, 1492. (贾江涛, 王蕾, 赵晴, 孙福兴, 朱广山, 化 学学报, 2013, 71, 1492.)

[16] Ma, L.; Abney, C.; Lin, W. Chem. Soc. Rev. 2009, 38, 1248.

[17] Dang, D.-B.; Wu, P.-Y.; He, C.; Xie, Z.; Duan, C.-Y. J. Am. Chem Soc. 2010, 132, 14321.

[18] Zhao, L.; Zeng, H.-P. Chin. J. Org. Chem. 2012, 32, 1633. (赵莉, 曾和平, 有机化学, 2012, 32, 1633.)

[19] Lan, Y.-Q.; Jiang, H.-L.; Li, S.-L.; Xu, Q. Adv. Mater. 2011, 23, 5015.

[20] Yan, A.-X.; Yao, S.; Li, Y.-G.; Zhang, Z.-M.; Lu, Y.; Chen, W.-L.; Wang, E.-B. Chem. Eur. J. 2014, 20, 6927.

[21] Dai, M.; Su, X.-R.; Wang, X.; Wu, B.; Ren, Z.-G.; Zhou, X.; Lang, J.-P. Cryst. Growth Des. 2014, 14, 240.

[22] Hou, Y.-L.; Sun, R.-W.; Zhou, X.-P.; Wang, J.-H.; Li, D. Chem. Commun. 2014, 50, 2295.

[23] Qin, J.-S.; Zhang, S.-R.; Du, D.-Y.; Shen, P.; Bao, S.-J.; Lan, Y.-Q.; Su, Z.-M. Chem. Eur. J. 2014, 20, 5625.

[24] Zhu, Y.; Wang, Y.-M.; Zhao, S.-Y.; Liu, P.; Wei, C.; Wu, Y.-L.; Xia, C.-K.; Xie, J.-M. Inorg. Chem. 2014, 53, 7692.

[25] Tan, Y.-X.; He, Y.-P.; Wang, M.; Zhang, J. RSC Adv. 2014, 4, 1480.

[26] Jayaramulu, K.; Reddy, S. K.; Hazra, A.; Balasubramanian, S.; Maji, T. K. Inorg. Chem. 2012, 51, 7103.

[27] Goswami, A.; Bala, S.; Pachfule, P.; Mondal, R. Cryst. Growth Des. 2013, 13, 5487

[28] Liu, Q.; Jin, L.-N.; Sun, W.-Y. CrystEngComm 2013, 15, 8250. 